\title{
ON PROJECTIVE SPHERES AND FUBINI AND TZITZEICA-WILCZYNSKI PSEUDOSPHERES
}

\author{
FROIM MARCUS
}

ABstract. The definition of a projective pseudosphere is given, and the following results are proved:

(1) There exists one and only one Fubini pseudosphere iff

$$
L=M=-\frac{3 \varphi^{2}}{2} . \quad \beta=\gamma=\varphi ; \quad \varphi=\varphi(\tau) \quad(\tau=u+v) .
$$

(2) There exist two classes of limiting Tzitzeica-Wilczymski pseudospheres or improper affine spheres.

These pseudospheres admit, beside the first directrix or affine normal, the first Fubini principal straight line or the conjugate of the first directrix with respect to the canonical tangent and projective normal. The asymptotes of the pseudosphere are twisted cubics.

1. Introduction. As a generalization of the metric definition of a sphere, E. Cartan [4] applies the term "projective sphere" to an unruled surface whose Fubini projective normal $[\mathbf{1}]$ passes through a fixed point. In projective differential geometry, every point of an unruled surface is associated with a pencil of straight lines projectively-connected with the surface. (These are named canonical lines or normals, and their plane is the canonical plane.) Thus there is a multiplicity of such spheres, each with its specific normal as above. The problem of determining surfaces of this kind is treated in $\S 28$ of $[\mathbf{1}]$ and was solved by Cech for the case of the axis passing through a fixed point. Surfaces of this kind depend generally on four constants. As regards the Fubini spheres, all that is known of them is that they depend on three arbitrary functions $[\mathbf{1}]$. Wilczynski $[\mathbf{2}]$ was the first to study a third variant--surfaces with indeterminate directrix curves (the first directrix, subsequently characterized as a canonical line [1], passing through a fixed point). The latter are the affine-geometry equivalent of Tzitzeica's $S$-surfaces, referred to by Blaschke [3] as "Affinspharen" and called here "Tzitzeica-Wilczynski surfaces".

The problem was treated by J. Kaucky $[\mathbf{5}, \mathbf{6}]$ and the solution was completed recently in $[\mathbf{7}-\mathbf{9}] .{ }^{1}$ We proved that (i) a projectively undeformable rotation surface can admit a canonical line passing through a fixed point, and (ii) a surface can admit at most two canonical lines or normals passing through fixed points. Consequently, we call projective spheres the unruled surfaces which admit one and only one normal or canonical line through a fixed point, and projective pseudospheres--those which admit two. They are determined by $[\mathbf{8}]$ :

$$
\beta=\gamma=-\frac{2}{c(u+v)} ; \quad L=M=-\frac{6}{c^{2}(u+v)^{2}},
$$

Received by the editors March 30, 1982.

1980 Mathematics Subject Classification. Primary 53A20.

${ }^{1}$ The following correction is in order on p. 347 of this reference: Cases $A=0, A=-1$ (not $A=1$ !) must be excluded. 
where $c$ is a solution of the algebraic equation

$$
(k+1)(k+3) c^{2}+4 k c-12=0,
$$

with

$$
(k+1)(k+3) \neq 0,
$$

where

$$
k=-3(1+2 \lambda) \text {. }
$$

In this paper, we attempt to find a class of Fubini spheres and prove that there exist one and only one Fubini pseudosphere and a class of limiting Tzitzeica-Wilczynski spheres, admitting (besides the first directrix) another normal through a fixed point.

2. Fubini spheres. Let $x(u, v)$ be an unruled surface with $u, v$ as asymptotic parameters. The straight line $(d)$ which joins a generic point $x$ with the point

$$
x_{u v}-l_{1} x_{v}-l_{2} x_{u}
$$

where .

$$
\begin{aligned}
& -l_{1}=\lambda \frac{\partial \log \beta \gamma^{2}}{\partial u}+\frac{1}{2}\left(\frac{\partial \log \beta \gamma}{\partial u}-\theta_{u}\right) ; \\
& -l_{2}=\lambda \frac{\partial \log \beta^{2} \gamma}{\partial v}+\frac{1}{2}\left(\frac{\partial \log \beta \gamma}{\partial v}-\theta_{v}\right),
\end{aligned}
$$

and $\lambda$ a parameter, is a canonical line or a normal, $\lambda=0,-1 / 2,-1 / 3, \infty$ represent, respectively, the projective normal, the directrix, the axis and the canonical tangent. Supposing that $x(u, v)$ is determined by Fubini's canonical system

$$
x_{u u}=\theta_{u} x_{u}+\beta x_{v}+p_{11} x ; \quad x_{v v}=\gamma x_{u}+\theta_{v} x_{v}+p_{22} x \quad(\theta=\log \beta \gamma) .
$$

then

$$
-l_{1}=\lambda \frac{\partial \log \beta \gamma^{2}}{\partial u} ; \quad-l_{2}=\lambda \frac{\partial \log \beta^{2} \gamma}{\partial v} .
$$

The developables of the congruence generated by the canonical line $(d)$ are $\left[\mathbf{1}^{\prime}\right]$ :

$$
\begin{aligned}
\left(\pi_{11}-l_{1 u}+l_{1} \theta_{u}-\beta l_{2}-l_{1}^{2}\right) d u^{2}+\left(l_{2 u}-l_{1 v}\right) d u d v & \\
& +\left(-\pi_{22}+l_{2 v}-l_{2} \theta_{v}+\gamma l_{1}+l_{2}^{2}\right)=0 .
\end{aligned}
$$

Hence $(d)$ passes through a fixed point if

(2.6) $\pi_{11}-l_{1 u}+l_{1} \theta_{u}-\beta l_{2}-l_{1}^{2}=0 ; \quad \pi_{22}-l_{2 v}+l_{2} \theta_{v}-\gamma l_{1}-l_{2}^{2}=0 ; \quad l_{2 u}=l_{1 v}$.

If surface $(x)$ is projectively deformable, then one passes to one of his deformates $(\bar{x})$ by changing only $\pi_{11}$ and $\pi_{22}$. Hence if conditions $(2.6)$ are verified for $(x)$ he cannot subsist for $(\bar{x})$. Therefore if a projective deformable surface admits a canonical line $(d)$ passing through a fixed point, all his deformates cannot admit a same canonical line $(d)$ passing through a fixed point.

If $(d)$ is the projective normal, then

$$
l_{1}=l_{2}=0
$$

and by $(2.6)$

$$
\pi_{11}=p_{11}+\beta_{v}+\beta \theta_{v}=0 ; \quad \pi_{22}=p_{22}+\gamma_{u}+\gamma \theta_{u}=0 .
$$


Supposing next that $x(u, v)$ is an isothermal-asymptotic surface, we can set $\beta=\gamma$, $(\theta=2 \log \beta)$, whence

$$
p_{11}=-3 \beta_{v} ; \quad p_{22}=-3 \beta_{u}
$$

From $\left[\mathbf{1}, \mathbf{1}^{\prime}\right]$ we have

$$
L=\theta_{u u}-\frac{1}{2} \theta_{u}^{2}-\beta_{v}-\beta \theta_{v}-2 p_{11} ; \quad M=\theta_{v v}-\frac{1}{2} \theta_{v}^{2}-\gamma_{u}-\gamma \theta_{u}-2 p_{22},
$$

and by $(2.8)$

$$
L-2(\log \beta)_{u u}+2(\log \beta)_{u}^{2}=3 \beta_{v} ; \quad M-2(\log \beta)_{v v}+2(\log \beta)_{v}^{2}=3 \beta_{u} .
$$

The integrability conditions of $(2.3)$ are then $[\mathbf{1}]$ :

$$
\begin{aligned}
& 3 \beta_{u u}-4 \frac{\beta_{v}}{\beta} \frac{\partial^{2} \log \beta}{\partial u \partial v}+3 \beta \beta_{v}=-2\left(\frac{\beta_{u}}{\beta}\right)_{v v}, \\
& 3 \beta_{v v}-4 \frac{\beta_{u}}{\beta} \frac{\partial^{2} \log \beta}{\partial u \partial v}+3 \beta \beta_{u}=-2\left(\frac{\beta_{v}}{\beta}\right)_{u u},
\end{aligned}
$$

which explains the difficulty in finding the spheres, even in the case of isothermal asymptotes. Operating with $\partial / \partial u$ of the first and with $\partial / \partial v$ of the second equation of $(2.11)$, we obtain

$$
\beta \beta_{u u u}-2 \beta_{u} \beta_{u u}=\beta \beta_{v v v}-2 \beta_{v} \beta_{v v} \quad(\beta \neq \text { cons. })
$$

which is identically satisfied if

$$
\beta=\varphi(\tau) \cdot(\tau=u+v) .
$$

In this case it follows from Fubini's form of the integrability conditions of $(2.3)[\mathbf{1}]$, that

$$
L=-\frac{3 \varphi^{2}}{2}+U(u) ; \quad M=-\frac{3 \varphi^{2}}{2}+V(v)
$$

with

$$
2 \varphi^{\prime}(V-U)+\varphi\left(U^{\prime}-V^{\prime}\right)=0
$$

Taking into account (2.8) and (2.10) we have

$$
U=V=C,
$$

whence

$$
L=M=-\frac{3}{2} \varphi^{2}+C
$$

Different values of $C$ represent projective rotation surfaces projectively applicable among themselves. The canonical curves of a surface (i.e., its intersections with the canonical planes) are determined by the differential equation

$$
\psi_{1} d u-\psi_{2} d v=0
$$

where

$$
\psi_{1}=\left(\log \beta \gamma^{2}\right)_{u} ; \quad \psi_{2}=\left(\log \beta^{2} \gamma\right)_{v}
$$


and if $\beta=\beta(\tau)$

$$
d u-d v=0,
$$

which yields a family of Segre curves. We prove that the Darboux curves

$$
d u+d v=0,
$$

are also plane, and that the conjugate net

$$
d u^{2}-d v^{2}=0
$$

is a Peterson net $[\mathbf{1 0}]$. The canonical planes then form a pencil whose axis is a fixed straight line. Hence the canonical lines intersect a fixed axis, and only the projective normals pass through a fixed point. According to the observation above, one and only one of the rotation surfaces given by (A) and (B) could admit Fubini's normal passing through a fixed point. From conditions (2.11) follows:

$$
\frac{2 \varphi^{\prime \prime \prime}}{\varphi}+3 \varphi^{\prime \prime}-\frac{10 \varphi^{\prime} \varphi^{\prime \prime}}{\varphi^{2}}+8\left(\frac{\varphi^{\prime}}{\varphi}\right)^{3}+3 \varphi \varphi^{\prime}=0,
$$

and integrating yields

$$
2 \frac{\varphi^{\prime \prime}}{\varphi}-4\left(\frac{\varphi^{\prime}}{\varphi}\right)^{2}+\frac{3}{2} \varphi^{2}+3 \varphi^{\prime}=C,
$$

which can be identified with (2.10) taking into account $\left(\mathrm{B}^{\prime}\right)$.

The above observation has general validity. Operating with $\partial / \partial u$ of the first and with $\partial / \partial v$ of the second equation of $(2.10)$, we obtain, respectively, the second and first conditions of (2.11).

To find the Fubini spheres which are projective rotation surfaces supposing $C=$ 0 , we must integrate the differential equation $\left(2.10^{\prime}\right)$ which now reads

$$
\varphi^{\prime \prime}-\frac{2\left(\varphi^{\prime}\right)^{2}}{\varphi}+\frac{3}{2} \varphi \varphi^{\prime}+\frac{3}{4} \varphi^{3}+0 .
$$

Denoting $p=\varphi^{\prime}$ and $\dot{p}=d p / d \varphi$ we have by $\left(2.10^{\prime}\right)$

$$
p \dot{p}-2 \frac{p^{2}}{\varphi}+\frac{3}{2} p \varphi+\frac{3}{4} \varphi^{3}=0
$$

which is an Abel differential equation of the second kind [11]. Setting

$$
p=\varphi^{2} u, \quad u=u(\varphi)
$$

we obtain

$$
u^{\prime}=\frac{d u}{d \varphi}=-\frac{3}{4}\left(\frac{1+2 u}{u \varphi}\right)
$$

For

$$
1+2 u \neq 0
$$

we have

$$
1+2 u=a \varphi^{3} \exp (2 u) \quad(a=\text { const. })
$$


whence the differential equation

$$
\varphi^{3} \exp \left(\frac{2 \varphi^{\prime}}{\varphi^{2}}\right)=\frac{\varphi^{2}+2 \varphi^{\prime}}{\varphi^{2}} .
$$

For

$$
1+2 u=0
$$

the solution reads

$$
\varphi=\frac{2}{\tau} \cdot(\tau=u+v)
$$

whence the following result:

To every solution of $(\delta)$, and to $\left(\delta^{\prime}\right)$, there corresponds a Fubini sphere which is a projective rotation surface.

We shall show below that the surface represented by the solution $\beta=\varphi=2 / \tau$ is a pseudosphere, in fact the only Fubini pseudosphere in existence if $C=0$.

Now let $C \neq 0$ and $\bar{\varphi}(\tau)$ be the solution of $\left(2.10^{\prime}\right)$. Setting $p=\bar{\varphi}^{\prime},\left(2.10^{\prime}\right)$ yields

$$
p \dot{p}+\frac{2 p^{2}}{\bar{\varphi}}-\frac{3}{2} \bar{\varphi} p+\frac{3}{4}\left(K-\bar{\varphi}^{3}\right) \quad\left(K=\frac{4}{3} C\right)
$$

which can be reduced to

$$
u u^{\prime}=\frac{3}{4}\left(K-(1+2 u) \bar{\varphi}^{3}\right)
$$

by putting $p=\bar{\varphi}^{2} u$.

It seems that the solution cannot be expressed explicitly. Therefore we consider in that which follows only the case $C=0$.

3. Fubini pseudospheres. Let us consider the existence problem of Fubini pseudospheres. The problem consists in finding spheres which admit, beside the projective normal, another canonical line $(d)$ passing through a fixed point. In this case we deal with the axis of the pencil of canonical planes, i.e. with a pair of fixed points. Supposing that $\lambda \neq 0$ represents the canonical line, then by $(2.4)$

$$
-l_{1}=3 \lambda \frac{\partial \log \beta}{\partial u} ; \quad-l_{2}=3 \lambda \frac{\partial \log \beta}{\partial u} .
$$

Noting that $\pi_{11}=\pi_{22}=0$ and $\beta=\varphi(\tau)$, we obtain from $\left(2.10^{\prime \prime}\right)$ and $(2.6)$ the set

$$
\begin{aligned}
& \frac{\varphi^{\prime \prime}}{\varphi}-2\left(\frac{\varphi^{\prime}}{\varphi}\right)^{2}+\frac{3}{2} \varphi^{\prime}+\frac{3}{4} \varphi^{2}=0 \\
& \frac{\varphi^{\prime \prime}}{\varphi}-(3 \lambda+3)\left(\frac{\varphi^{\prime}}{\varphi}\right)^{2}+\varphi^{\prime}=0 .
\end{aligned}
$$

Our problem thus reduces to finding the common solution of this set, which evidently depends on $\lambda$. We shall prove that the set admits a unique solution. Indeed, it follows from $(D)$ by subtraction that

$$
(3 \lambda+1)\left(\frac{\varphi^{\prime}}{\varphi}\right)^{2}+\frac{1}{2} \varphi^{\prime}+\frac{3}{4} \varphi^{2}=0 .
$$


It can be proved that $(d)$ cannot be the axis. If it were, we would have $1+3 \lambda=0$, and could without loss of generality take the solution

$$
\varphi=\frac{2}{3 \tau} \quad(\tau=u+v)
$$

which does not satisfy the set. Accordingly, let

$$
1+3 \lambda=\alpha \quad(\alpha \neq 0)
$$

and (3.2) yields by integration

$$
\varphi=-\frac{1}{A \tau}
$$

where

$$
A=\frac{-1 \pm \sqrt{1-12 \alpha}}{4 \alpha}
$$

The set (D) yields for the two values of $A$

$$
\alpha=-2 ; \quad A=-\frac{1}{2} .
$$

The common solution reads

$$
\varphi=\frac{2}{\tau},
$$

and from (3.7) and (3.4) it follows that

$$
\lambda=-1 \text {. }
$$

This is a new canonical line or normal which apparently was not considered so far. Thus we have the following result:

There exists one and only one Fubini pseudosphere, given by

$$
\beta=\lambda=\frac{2}{\tau} ; \quad L=M=-\frac{6}{\tau^{2}} \quad(\tau=u+v) .
$$

4. Tzitzeica-Wilczynski pseudospheres. Tzitzeica-Wilczynski spheres are obtained from the set

$$
x_{u u}=\theta_{u} x_{u}+\beta x_{v} ; \quad x_{v v}=\beta x_{u}+\theta_{v} x_{v} ; \quad \theta_{u}=-\frac{\beta_{u}}{\beta} ; \quad \theta_{v}=-\frac{\beta_{v}}{\beta},
$$

which is completely integrable if

$$
\frac{\partial^{2} \log \beta}{\partial u \partial v}=\beta^{2}+k \beta \quad(k=\text { const. })
$$

and the first Wilczynski directrix, represented by $\lambda \doteq-\frac{1}{2}$, passes through a fixed point.

For $k \neq 0$ they are Tzitzeica spheres or, in affine-geometry, proper affine spheres $[3]$, the directrix being the affine normal. Suppose first $k \neq 0$; by appropriate change of the parameters $u, v, k$ can be set at 1 . Hence

$$
\frac{\partial^{2} \log \beta}{\theta u \partial v}=\beta^{2}+\frac{1}{\beta}
$$


Suppose next that the surface admits a canonical line $(d)$ other than the directrix, i.e. $\lambda \neq-\frac{1}{2}$. From (4.1) and (2.2) it follows that

$$
-l_{1}=3\left(\lambda+\frac{1}{2}\right)(\log \beta)_{u} ; \quad-l_{2}=3\left(\lambda+\frac{1}{2}\right)(\log \beta)_{v} .
$$

Bearing in mind $(2.7),(2.6)$ and $(4.1)$, the condition for $(d)$ passing through a fixed point reads:

$$
(\log \beta)_{u u}=\left(3 \lambda+\frac{1}{2}\right)\left(\frac{\beta_{v}}{\beta}\right)-\beta_{v} ; \quad(\log \beta)_{v v}=\left(3 \lambda+\frac{1}{2}\right)\left(\frac{\beta_{v}}{\beta}\right)^{2}-\beta_{u}
$$

whence

$$
\begin{aligned}
& (\log \beta)_{u u v}=2\left(3 \lambda+\frac{1}{2}\right)(\log \beta)_{u}(\log \beta)_{u v}-\beta_{v v} \\
& (\log \beta)_{v v u}=2\left(3 \lambda+\frac{1}{2}\right)(\log \beta)_{v}(\log \beta)_{v u}-\beta_{u u}
\end{aligned}
$$

which, on account of the partial derivatives in (4.3), yields in turn

$$
\begin{aligned}
& 2 \beta_{u}\left(1+3 \lambda+3 \lambda \beta^{3}\right)=3\left(\lambda+\frac{1}{2}\right) \beta \beta_{v}^{2}, \\
& 2 \beta_{v}\left(1+3 \lambda\left(1+\beta^{3}\right)\right)=3\left(\lambda+\frac{1}{2}\right) \beta \beta_{u}^{2} .
\end{aligned}
$$

Consequently, the condition for a Tzitzeica-Wilczynski surface admitting two canonical normal lines through fixed points reads

$$
\beta_{u}^{3}=\beta_{v}^{3} .
$$

For comparison with the results of $[\mathbf{5}]$, we can take without loss of generality

$$
\beta=\beta(\tau), \quad \tau=u+v .
$$

By (4.3) we have

$$
\frac{\varphi^{\prime \prime}}{\varphi}-\left(\frac{\varphi^{\prime}}{\varphi}\right)^{2}-\varphi^{2}-\frac{1}{\varphi}=0
$$

while by (4.5)

$$
\frac{\varphi^{\prime \prime}}{\varphi}-3\left(\lambda+\frac{1}{2}\right)\left(\frac{\varphi^{\prime}}{\varphi}\right)^{2}+\varphi^{\prime}=0 \quad\left(\lambda+\frac{1}{2} \neq 0\right)
$$

and the common solution of the set (4.8)--(4.9) determines the corresponding pseudospheres.

5. Limiting Tzitzeica-Wilczynski pseudospheres. Limiting TzitzeicaWilczynski spheres are determined by the set (4.1) and the condition

$$
\frac{\partial^{2} \log \beta}{\partial u \partial v}=\beta^{2} \text {. }
$$

To obtain the corresponding pseudospheres, we must integrate the set obtained from (5.1) and (4.5) namely

$$
\frac{\varphi^{\prime \prime}}{\varphi}-\left(\frac{\varphi^{\prime}}{\varphi}\right)^{2}-\varphi^{2}=0 ; \quad \frac{\varphi^{\prime \prime}}{\varphi}-3\left(\lambda+\frac{1}{2}\right)\left(\frac{\varphi^{\prime}}{\varphi}\right)^{2}+\varphi^{\prime}+0 \quad\left(\lambda+\frac{1}{2} \neq 0\right)
$$


By these,

$$
\left(3 \lambda+\frac{1}{2}\right)\left(\varphi^{\prime}\right)^{2}-\varphi^{\prime} \varphi^{2}-\varphi^{4}=0 .
$$

We observe that iff

$$
3 \lambda+1 / 2=0
$$

i.e.

$$
\lambda=-1 / 6
$$

the solution

$$
\varphi=1 / \tau,
$$

satisfies the set (5.2), and $(d)$ is the first Fubini principal straight line [1]. Suppose now that

$$
3 \lambda+1 / 2=\alpha \neq 0 .
$$

Then from (5.3) it follows that

$$
\varphi=-1 / A \tau
$$

where

$$
A=\frac{1 \pm \sqrt{1+4 \alpha}}{2 \alpha} .
$$

From the set (5.2), we deduce $A=1, \alpha=2$, hence the solution is

$$
\varphi=1 / \tau \text {. }
$$

At the same time, for $\alpha=2$

$$
\lambda=1 / 2 .
$$

Hence the second canonical line is the conjugate normal of the first directrix with respect to the projective normal and canonical tangent, and we have the following result:

Limiting Tzitzeica-Wilczynski pseudospheres (or "improper affine spheres") admit (beside the first directrix or affine normal) a second normal passing through a fixed point. The latter is readily shown to be at infinity.

6. Another interpretation. It is well known that the limiting TzitzeicaWilczynski surfaces are represented in finite form by ${ }^{2}$

(6.1) $x_{1}=(u-v)\left(U^{\prime}-V^{\prime}\right)-2(U-V) ; \quad x_{2}=U^{\prime}-V^{\prime} ; \quad x_{3}=u+v ; \quad x_{4}=1$,

where $U, V$ are, respectively, arbitrary functions of $u$ and $v$, and $U^{(3)} V^{(3)} \neq 0$. From (6.1), it follows that

$$
\beta=-\frac{U^{(3)}}{\sigma} ; \quad \gamma=-\frac{V^{(3)}}{\sigma} ; \quad \sigma=U^{\prime \prime}+V^{\prime \prime} ; \quad \theta_{u}=-\beta ; \quad \theta_{v}=-\gamma .
$$

\footnotetext{
him.

${ }^{2}$ This representation was obtained by Wilczynski $[\mathbf{2}]$ and is repeated in $[\mathbf{3}]$ without credit to
} 
Suppose now that the surfaces admit a $G_{2}$ group of projective deformations into themselves, generated by the i.t.

$$
X_{1}=\frac{\partial}{\partial u}-\frac{\partial}{\partial v} ; \quad X_{2}=u \frac{\partial}{\partial u}+v \frac{\partial}{\partial v} .
$$

Then, $U$ and $V$ are given by

$$
U=a u^{3}+a_{1} u^{2}+a_{2} u+a_{3} ; \quad V=a v^{3}-a_{1} v^{2}+b_{2} v+b_{3},
$$

whence

$$
\beta=\gamma-\frac{1}{u+v} ; \quad L=M=-\frac{3}{2(u+v)^{2}},
$$

and the infinitesimal transformation is projective [12]. From (4.5), it follows that

$$
\lambda=1 / 2 \text {. }
$$

If the $G_{2}$ group generated by the i.t.

$$
X_{1}=\frac{\partial}{\partial u}+\frac{\partial}{\partial v} ; \quad X_{2}=u \frac{\partial}{\partial u}+v \frac{\partial}{\partial v} .
$$

Then,

$$
\begin{aligned}
& U=a u^{3}+a_{1} u^{2}+a_{2} u+a_{3} ; \quad V=-a v^{3}-a_{1} v^{2}+b_{2} v+b_{3}, \\
& \beta=\gamma-\frac{1}{u-v} ; \quad L=M=-\frac{3}{2(u-v)^{2}}
\end{aligned}
$$

and by the same conditions we obtain the normal determined by

$$
\lambda=-1 / 6 \text {. }
$$

In all these cases the asymptotes are twisted cubics (see [12]).

\section{BIBLIOGRAPHY}

1. G. Fubini and E. Cech, Geometria proiettiva differenziale, vol. 1, Zanichelli, Bologna, 1926.

$1^{\prime}$. __ Introduction à la géométrie projective differentielle des surfaces, Gauthier-Villars, Paris, 1931.

2. E. Y. Wilczynski, Über Flächen mit unbestimten Direktrixkurven, Math. Ann. 76 (1915), 129-160.

3. W. Blaschke, Differential geometrie, Springer-Verlag, Berlin, 1923, pp. 212, 216.

4. E. Cartan, Sur la connexion projective des surfaces, C. R. Acad. Sci. Paris Ser. A B 178 (1929), 750 .

5. J. Kaucky, Étude des surfaces dont une droite canonique passe par un point fixe, Publ. No. 109 de la Faculté des Sci. de l'Université Masaryk, Brno.

6. __ Sur les surfaces dont une droite canonique passe par un point fixe, Rend. Accad. Leincei 9 (1929).

7. F. Marcus, On the results of J. Kaucky concerning the problem of the determination of surfaces for which a canonical line passes through a fuxed point, Acad. Roy. Belg. Cl. Sci. 63 (1977), 274-282.

8. Completion of Cech's and Kaucky's results of surfaces on which a canonical line passes through a fixed point, Ann. Mat. Pura Appl. (4) 114 (1977), 319-330.

9. __ Sur les surfaces dont deux normales ou droites canoniques passent par un point fuxe, An. Ştiinţ. Univ. "Al. I. Cuza" Iaşi Sect. Ia Mat. (N.S.) 24 (1978), 345-349.

10. __ Sur les reseaux de Koenigs, Rev. Mat. Pures Appl. 2 (1957), 555-559.

11. E. Kamke, Differentialgleichungen 3 Auflage, Chelsea, New York, pp. 26-28.

12. F. Marcus, Again on the surfaces which allow $\infty^{2}$ projective transformations into themselves, An. Ştịinţ. Univ. "Al. I. Cuza" Iaşi Sect. Ia Mat. (N.S.) 22 (1976).

Department of Mathematics, Technion-Israel institute of Technology, TEChNion City, Haifa, 32000, ISRAEL 